\title{
BioLogger: A Wireless Physiological Sensing and Logging System with Applications in Poultry Science
}

\author{
Sheng $\mathrm{Hu}$ and Jindong Tan
}

\begin{abstract}
This paper presents the design and development of BioLogger, a wireless physiological signal sensing and logging system. BioLogger can simultaneously monitor and record various types of physiological signals. Energy saving design is incorporated to both hardware and software design phases in order to prolong the life time of sensor nodes. Moreover, a simple scheduler is implemented to make sure the emergency would not be missed.
\end{abstract}

\section{INTRODUCTION}

Monitoring of physiological signals in free living environments is not only important humans but also animal related researches. For instance, recent poultry science research interests fear-related behaviors of chicken in both cage and cage-free environments. During the controlled atmosphere stunning of chickens in the automated live hang processes at broiler processing plants, monitoring of the key physiological signals allow inferences to be drawn at the time to loss of consciousness, time to non-recovery [1]. Signals will be evaluated for the purpose of animal welfare and nutrition maintaining. Meanwhile, more and more health-care services rely on the patient's vital signs to maintain his optimal health status and improve the accuracy of the diagnosis and the therapy. The requirements from these domains necessitate the development of wireless physiological monitoring and recording systems.

The use of wearable wireless sensors for the monitoring of physiological signals attracts more and more attentions. A traditional monitoring system usually uses a direct-wired connection between the subject and the instrument, the subject is therefore confined to the monitoring instrument. However a wireless monitoring system detaches the sensing unit from the processing unit. Hence the subject with wearable sensors can be monitored in a free living environment. A conventional monitoring system is usually only used for collecting data, but data processing and diagnosis are done offline [2]. In contrast, the on-body sensor node in the wireless monitoring system can detect early abnormities by local computational capacity, even the subject is unaware and unconscious [3]. For the aging people with heart deceases, emergencies often occur when the patients are falling asleep. Hence it is significant that the medical disorders can be detected early. Finally, using the telecommunication infrastructure [4], signals can be directly delivered to the clinics, where doctors can remotely analyze real-time physiological status of the subject, and make correct diagnosis timely.

Sheng $\mathrm{Hu}$ and Jindong Tan are with the Department of Electrical and Computer Engineering, Michigan Technological University, Houghton, MI 49931, USA \{shengh, jitan\}@mtu.edu
There exist technical challenges for the application of wearable wireless sensors in biology science and healthcare. Firstly, most existing wireless monitoring systems only consider monitoring single physiological signal [5], [6]. Sometimes, multiple physiological status shall be monitored simultaneously. For example, a subject's respiration rate is detected higher than normal, it is possible that the subject is having exercise at that time, or there are possibilities that the subject is suffering heart attacks. Secondly, energy efficiency of the software and hardware design is critical for long term monitoring with wearable battery-powered sensors.

To address these technical challenges, BioLogger, a platform prototype for biological monitoring and logging, is proposed in this paper. This system is able to sense the various real-time physiological signals simultaneously: electrocardiogram (ECG), electroencephalogram (EEG), respiration rate and skin temperature. Moreover, an extension socket is for additional signals. Energy efficiency considerations of BioLogger include low power hardware and software design, and power efficient networking. All the components in BioLogger are carefully chosen with the sleep mode, so that they could be configured into sleeping mode according to the requirements of applications. Additinally, a simple and low-cost network protocol is adopted in BioLogger to reduce the power consumption for data transmission. In the firmware of the wearable sensor, a simple real-time scheduler is used to guarantee scheduling of tasks. Normally, the scheduler maintains the periodic data acquisition and processing tasks in the system. When a physiological abnormality is detected, a sporadic task with firm deadline and higher priority is generated to handle the emergency.

\section{SYSTEM DESIGN}

BioLogger comprises three parts: multiple on-body Sensor Nodes, a Base Station and a Terminal Computer, as shown in Figure 1. Sensor Node (Figure 2(b)) attached to the subject's body acquires the physiological signals from the subject, and converts them to digital data. These data can be stored in MicroSD card on Sensor Node, preprocessed using the on-board computational capability, or transmitted to Base Station wirelessly.

Base Station relays those physiological data to Terminal Computer via a USB port. A graphic user interface(GUI) on Terminal Computer serves as a processing terminal, which real-time displays the digitalized signals according to user's demand, and store the raw data on the hard disk in ASCII format. 

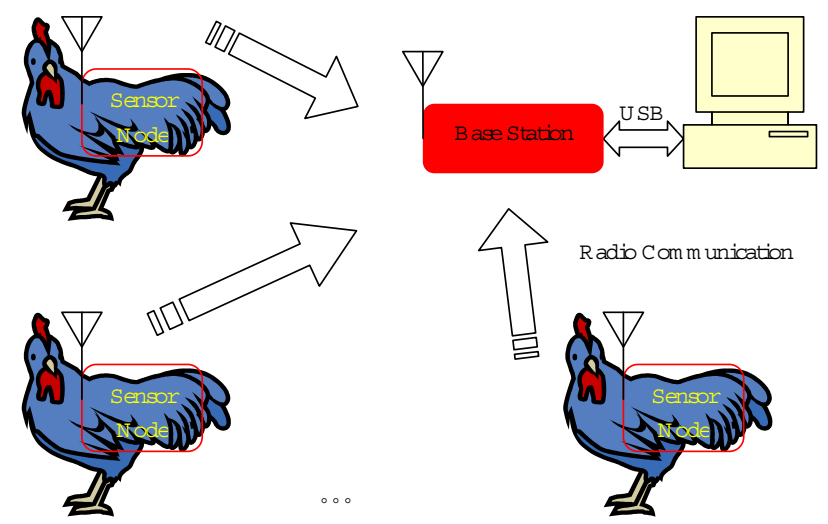

Fig. 1. BioLogger system architecture.

TABLE I

SPECIFICATIONS OF SENSOR NodE.

\begin{tabular}{llr}
\hline \hline Module & Parameter & Specification \\
\hline ECG & Gain & 120 \\
& Sampling rate & $100 \mathrm{~Hz}$ \\
& Low pass cutoff frequency & $40 \mathrm{~Hz}$ \\
& High pass cutoff frequency & $0.1 \mathrm{~Hz}$ \\
\hline EEG & Gain & 7680 \\
& Sampling rate & $100 \mathrm{~Hz}$ \\
& Low pass cutoff frequency & $30 \mathrm{~Hz}$ \\
& High pass cutoff frequency & $0.1 \mathrm{~Hz}$ \\
\hline Temperature & Sampling rate & $1 \mathrm{~Hz}$ \\
& Sensing Range & $150^{\circ} \mathrm{C}$ \\
\hline Respiration & Sampling rate & $10 \mathrm{~Hz}$ \\
\hline On-chip ADC & Resolution & $10 \mathrm{bit}$ \\
\hline Radio & Data rate & $250 \mathrm{Kbps}$ \\
\hline Storage & Capacity & $2 \mathrm{~GB}$ \\
\hline Power & Voltage & $3 \mathrm{~V}$ \\
\hline \hline
\end{tabular}

\section{A. Sensor Node}

Sensor Node consists of 7 modules: a microcontroller unit(MCU), a radio communication module, 4 conditioning circuits, and a storage module, as shown in Figure 2(a). The raw signals from the sensors are feeded into corresponding conditioning circuits for noise decoupling, and then they are amplified to a proper dynamic range for the on-chip analog-to-digital convertor (ADC). After a 15-channel ADC digitalizes the analog signals, MCU can process the data on the specific requirements. The storage module can save all the data on a MicroSD card and the radio module can transmit data wirelessly for monitoring. The specifications are summarized in Table I. The following subsections will discuss the modules in detail.

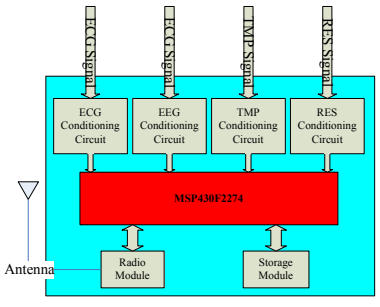

(a) Block diagram

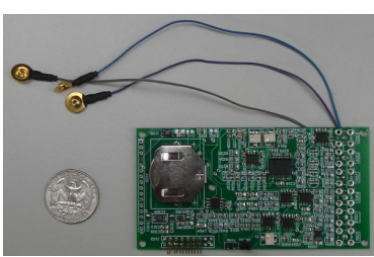

(b) A picture of Sensor Node
Fig. 2. The on-body Sensor Node.
1) Microcontroller Unit: MCU plays a critical role in Sensor Node. Sensor Node embeds a 16-bit RISC MCU, MSP430F2274. It is an ultra low-power MCU (Table II, especially targeted for the battery powered device. It contains 32KB Flash, 1KB RAM and rich integrated peripherals including a fast 10-bit ADC. MCUs from MSP430 family have been widely applied to various sensor networks (Tmote Sky[7] etc.), from which the previous reference design helps to cut the developing cost.

2) Wireless Network: The topology of BioLogger is a star-like network (Figure 1) based on SimpliciTI. SimpliciTI is a simple and low cost wireless network protocol, which supports up to $250 \mathrm{Kbps}$ data transmission rate and 4 hops in the wireless networks. Under SimpliciTI, only 5 command APIs can be used to configure the network's functionalities, and memory requirement only needs up to 4KB Flash and 512B RAM [8]. Compared to IEEE802.15.4, SimpliciTI has the relatively simple MAC frame: the overhead only contains 9 bytes, while IEEE802.15.4 [9] contains a 23byte header in its MAC sublayer. As shown in Table II, radio communication has higher order of power consumption than other parts, therefore a simple MAC sublayer will significantly improve transmission efficiency, and reduce the energy consumption correspondingly.

TABLE II

POWER CONSUMPTION OF THE PARTS IN BIOLOGGER.

\begin{tabular}{llr}
\hline Parts & Operating Status & Typical Current \\
\hline MSP430F2274 & Active Mode@ @ 1MHz & $390 \mu \mathrm{A}$ \\
& LPM3@1MHz & $0.5 \mu \mathrm{A}$ \\
\hline CC2500 [10] & Receiving Mode & $12.8 \mathrm{~mA}$ \\
& Transmitting Mode & $21.6 \mathrm{~mA}$ \\
& Sleep Mode & $400 \mathrm{nA}$ \\
\hline
\end{tabular}

3) ECG Conditioning Circuit: The unconditioned ECG from the electrode is in the order of microvolts and always coupled with large common mode noise. Moreover, the subject's body acts as a very strong antenna, which resonates at $60 \mathrm{~Hz}$, and consequently picks up the electrical noise on the order of several hundred millivolts. The ECG conditioning circuit (Figure 3) is designed to remove the interference of common mode noise and suppress $60 \mathrm{~Hz}$ electrical noise.

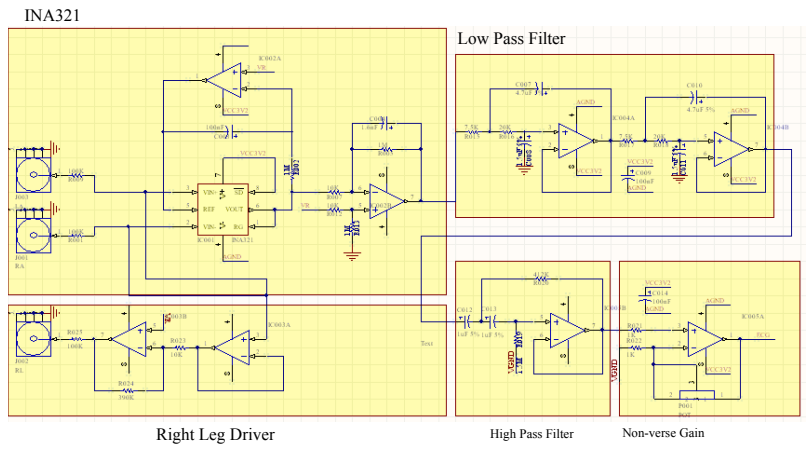

Fig. 3. ECG conditioning circuit.

An high common mode rejection ratio (94dB) instrumental amplifier, INA321, is integrated to the front end to reject the 
common mode. In addition, the right leg driver circuit is built to reduce common mode noise further by injecting the common mode signals back into the body to cancel them out [11].

Since the human's ECG falls in the frequency range between 10 to $30 \mathrm{~Hz}$, hence a fourth order Chebyshev low pass filter is followed to remove parasitic noise including $60 \mathrm{~Hz}$ electrical noise. The Chebyshev filter owns a steeper cutoff slope than other types of filters under the same complexity. At the end stage, a high pass filter is followed to remove the ultra low frequency drift and a non-inverting gain circuit to meet the dynamic range of ADC.

4) EEG Conditioning Circuit: Similar to ECG, EEG is also an analog signal with an ultra-small amplitude and low frequency and corrupted with big common mode noise; therefore the conditioning circuit of EEG follows the same pattern: one instrumental amplifier plus filters. However, EEG occurs at a different frequency band and even smaller amplitude (on the order of microvolts), thus the cutoff frequency of the filters should be setup at different point.

5) Respiration Conditioning Circuit: In BioLogger, a strip piezo film serves as a respiratory sensor. The voltage generated by the piezo film will decay once the exerted force does not change. This property is quantified by Time Constant, which represents the time required for a signal to decay to $70.7 \%(-3 \mathrm{~dB})$ of its original amplitude [12]. The frequency of adults' respiration rate normally ranges from 0.2 to 0.5 $\mathrm{Hz}$ [13]. Therefore, TimeConstant $=R 401 \times C_{\text {Piezo }}=0.1 \mathrm{~s}$ on Sensor Node to guarantee that the signal does not decay too fast, as shown in Figure 4. The RC network at the end of the circuit is to remove the coupled high frequency noise.

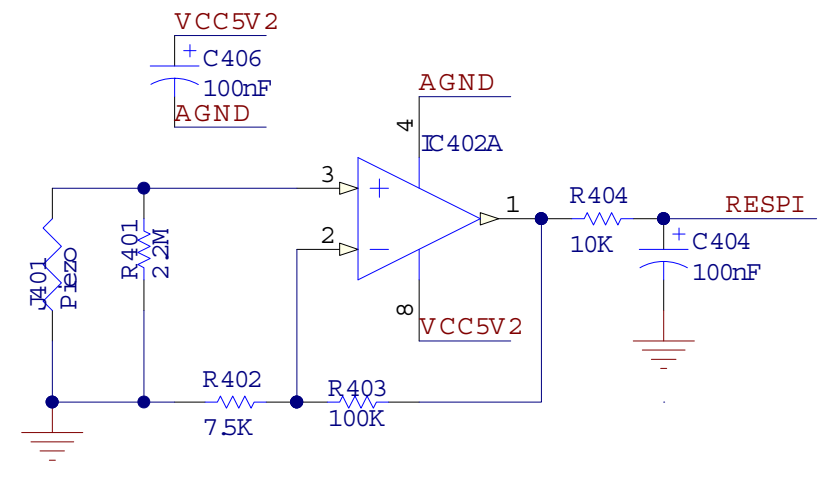

Fig. 4. Respiration conditioning circuit.

6) Temperature Conditioning Circuit: The temperature conditioning circuit mainly consists of a voltage divider, a voltage follower, and a RC network, as is shown in Figure 5. The voltage divider includes a temperature sensor and a resistor. $J 402$ is a $10 \mathrm{~K} 3 \mathrm{NTC}$ temperature sensor from Betatherm Ireland Ltd. Its $R / T$ relation follows $R_{T} \propto$ $\exp \left(\frac{1}{T}\right)$ [14], where $R_{T}$ is the resistance of the thermistor at a particular temperature $T$ (Unit: Kelvin). $R 407$ is a ultrasmall temperature coefficient resistor, whose resistance keeps invariant when the temperature changes. At the consequent two stages, a voltage follower decreases the output resistance, and the RC network decouples the high frequency noise. In the implementation, $V_{c c}$ is also sampled to compensate for its drift.

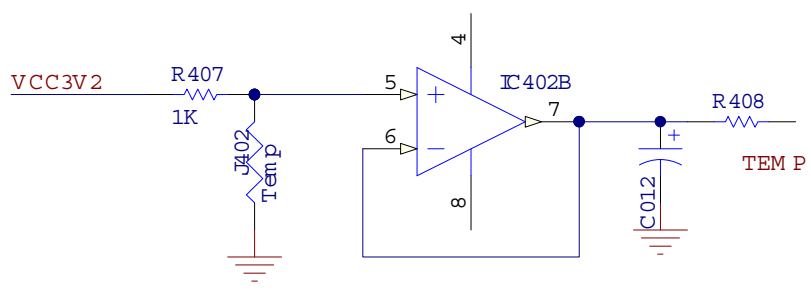

Fig. 5. Temperature conditioning circuit.

In order to convert the digitized ADC readings to temperature value, it is a necessary step to calibrate the sensor in advance. Sensor Node constructs a look-up table that contains all the values of temperature corresponding to the ADC readings, which will trade the time by on memory space. This is a acceptable option, because the flash memory on the MCU is large enough for the look-up table, but computation time is critical in the real-time monitoring system.

7) Firmware: A simple real-time task scheduler is implemented on Sensor Node to manage three periodic tasks: 1)acquiring physiological signals; 2) preprocessing the digitalized signals and 3) encoding and transmitting the data to Base Station. In addition, if some abnormalities are detected in the second task, a non-exemptible sporadic task with a higher priority is released to sound alarm. The abnormalities can be defined by the users in advance.

\section{B. Base Station and Terminal Computer}

Base Station relays the physiological data and alarm messages from Sensor Nodes to Terminal Computer. And a graphic user interface (GUI) on Terminal Computer displays the data visually and store them in a .txt file.

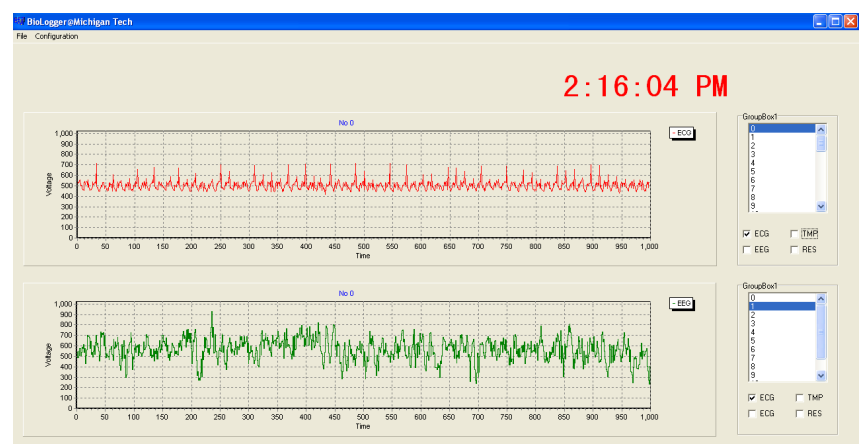

Fig. 6. The GUI on Terminal Computer.

\section{EXPERIMENTAL RESULTS}

We have successfully demonstrated functionalities of BioLogger on broiler hens (Figure 7(a)). Before the test, a three-lead EEG electrode was implanted in every hen, and hens are then recovered from the operation for four days. Two disposable, self-adhesive ECG electrodes were adhered 
to the cleaned skin overlying the hen's pectoralis muscle at either side of the sternum, and the right leg electrode was attached to the ham at the right hand side. In addition, saline water is applied on the cleaned skin where ECG electrodes locate to increase the electrical conductivity. A temperature sensor is adhered to the skin underneath the swing by tape. A piezo strip is tightly circled around the body of the bird, and its two ends are fixed on the chick's belly, where the respiration can be clearly sensed. Figure 7(b), Figure 7(c) and Figure 7(d) show the typical ECG, EEG and respiration waveforms of a hen.

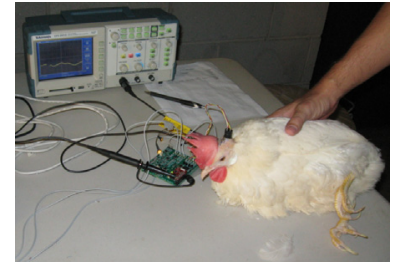

(a) Experimental setup

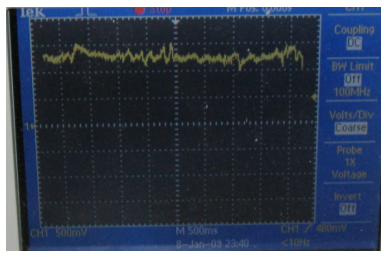

(c) EEG waveform

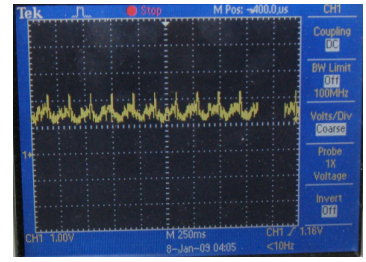

(b) ECG waveform

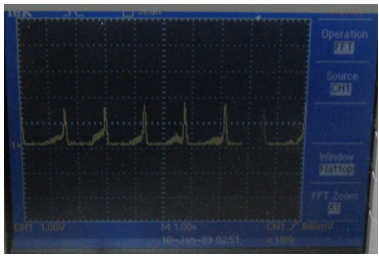

(d) Respiration waveform
Fig. 7. Typical physiological signals from a hen.

Another chicken welfare experiment was to observe the EEG waveform when a hen is placed in $\mathrm{CO}_{2}$ environment. All the EEG data were acquired by BioLogger and analyzed by Spike2 (as shown in Figure 8). The part of the waveform before the red line in the top chart shows the EEG of a relaxed hen. And at time point of the red line, we stimulated the bird for one time, the hen was alert and the EEG waveform pattern changed immediately from low to high frequency and from large to small amplitude. The top chart illustrates that the hen responded to external stimulations in terms of EEG. Then we placed the hen in $\mathrm{CO}_{2}$ environment for 15 seconds. The middle chart records the EEG waveform after the hen was taken out of $\mathrm{CO}_{2}$. At first, the bird was unconscious and then waked up. The EEG waveform of the wake-up moment is denoted by the blue line, as shown in the middle chart. Last, we placed the hen in $\mathrm{CO}_{2}$ environment till dead, the bottom chart shows the hen's EEG waveform after its death.

\section{COnClusion And Future Work}

BioLogger, a wireless physiological monitoring and logging system has been successfully implemented. BioLogger can simultaneously monitor and log various types of physiological signals including ECG, EEG, respiration and skin temperature. Energy efficient approaches are incorporated in both hardware and software design phases in order to prolong the lifetime of sensor nodes. Moreover, a simple real time

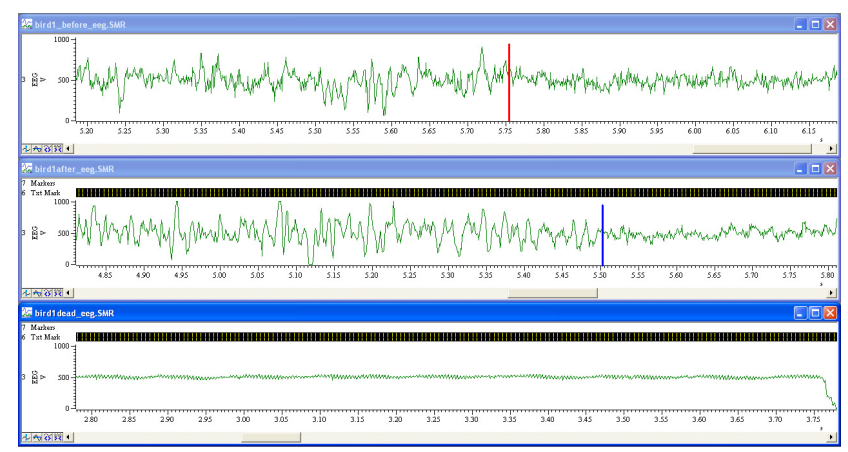

Fig. 8. EEG waveform in the welfare experiment.

scheduler is implemented to guarantee that the timeliness will not be violated in case of emergencies.

As part of future work, the size of Sensor Node will be reduced further, and the system will be extended to monitor more physiological signals. Ideally, BioLogger will be improved to provide the interface to a smart phone, and users can observe their own physiological status ambulatorily.

\section{REFERENCES}

[1] Alexei L. Vyssotski, Andrei N. Serkov, Pavel M. Itskov, Giacomo Dell'Omo, Alexander V. Latanov, David P. Wolfer, and Hans-Peter Lipp. Miniature neurologgers for flying pigeons: Multichannel eeg and action and field potentials in combination with gps recording. Journal of Neurophysiology, 95:1263-1273, 2006.

[2] Chris Otto, Aleksandar Milenkovic, corey sanders, and Emil Jovanov. System architectures of a wireless body area sensor network for ubiquitous health monitoring. Journal of Mobile Multimedia, 1:307326, 2006.

[3] Samuel Ng Choon Po, Guo Dagang, Mohammad Dzulkifli Bin Mohyi Hapipi, and Francis Tay Eng Hock. Memswear biomonitoring incorporating sensors into smart shirt for wireless sentinel medical detection and alarm. International MEMS Conference 2006, pages 1068-1072, 2006.

[4] David Malan, Thaddeus FulfordJones, Matt Welsh, and Steve Moulton. Codeblue: An ad hoc sensor network infrastructure for emergency medical care. In Proc of the MobiSys 2004 Workshop on Applications of Mobile Embedded Systems, pages 12-14, June 2004.

[5] Thaddeus R. F. Fulford-Jones, Gu-Yeon Wei, and Matt Welsh. A portable, low-power, wireless two-lead ekg system. Engineering in Medicine and Biology Society, 26th Annual International Conference of the IEEE, 1:2141-2144, Sept. 2004.

[6] Boqiang Liu, Yanyan Zhang, Zhongguo Liu, and Cong Yin. An embedded eeg analyzing system based on c/os-ii. Engineering in Medicine and Biology Society, 29th Annual International Conference of the IEEE, pages 2468-2471, Aug. 2007.

[7] Tmote sky datasheet. Technical report, Sentilla Corporation, 2006.

[8] Larry Friedman. Simpliciti: Simple modular rf network specification. Technical report, Texas Instruments, 2008.

[9] Robert F. Heile. Ieee standard 802.15.4. Technical report, IEEE Computer Society, 2003.

[10] Cc2500: Low-cost low-power 2.4 ghz rf transceiver. Technical report, Texas Instruments, 2007.

[11] Graham Bancroft, Neale Genereux, Rizwin Sheergar, Sumeet Goyal, Jacky Lin, and Ivan Chan. Eece 474/375 instrumentation and design laboratory final report. Technical report, Department of Electrical and Computer Engineering, 2006.

[12] Measurement Specialties, Inc. Piezo Film Sensors Technical Manual, rev b edition, April 1999.

[13] William D. McArdle, Frank I. Katch, and Victor L. Katch. Exercise Physiology: Energy, Nutrition, and Human Physiology. Lippincott Williams \& Wilkins, 2006.

[14] Pat Lyons and Phil Waterw. The use of ntc thermistors as sensing devices for tec controllers and temperature control integrated circuits. Technical report, Betatherm Ireland Ltd., 2003. 